\title{
New observations of asteroid (175706) 1996 FG3, primary target of the ESA Marco Polo-R mission
}

\author{
J. de León ${ }^{1}$, T. Mothé-Diniz ${ }^{2}$, J. Licandro ${ }^{3,4}$, N. Pinilla-Alonso ${ }^{5}$, and H. Campins ${ }^{6}$ \\ ${ }^{1}$ Instituto de Astrofísica de Andalucía-CSIC, Glorieta de la Astronomía, s/n. 18008 Granada, Spain \\ e-mail: jleon@iaa.es \\ 2 Universidade Federal do Rio de Janeiro/Observatório do Valongo Lad. Pero Antônio, 43 - 20080-90, Rio de Janeiro, Brazil \\ e-mail: thais.mothe@astro.ufrj.br \\ 3 Instituto de Astrofísica de Canarias (IAC), C/Vía Láctea s/n, 38205 La Laguna, Spain \\ e-mail: jlicandr@iac.es \\ 4 Departamento de Astrofísica, Universidad de La Laguna (ULL), 38205 La Laguna, Spain \\ 5 NASA Postdoctoral Program, Resident Research Associated at NASA Ames Research Center MS 245-3, Moffett Field, \\ CA 94035-1000, USA \\ ${ }^{6}$ University of Central Florida, PO Box 162385, Orlando, FL 32816.2385, USA
}

Received 6 April 2011 / Accepted 10 May 2011

ABSTRACT

\begin{abstract}
Context. Near-Earth asteroid (175706) 1996 FG3 is the primary target of the ESA Marco Polo-R mission, which was selected for the assessment study phase of ESA M3 missions. This is a primitive (C-type), binary asteroid that will allow new research to be performed. The primary is a rapidly rotating $(3.6 \mathrm{~h})$ small asteroid $(1.4 \mathrm{~km}$ diameter) that is almost spherical and has a satellite of $\sim 400 \mathrm{~m}$.

Aims. We analyse new ground-based spectroscopic data of 1996 FG3 to help us characterise its surface composition and prepare for the mission.

Methods. We obtained a near-infrared spectrum covering the range $0.8-2.5 \mu \mathrm{m}$, using the camera-spectrograph NICS at the $3.6 \mathrm{~m}$ telescope TNG (Telescopio Nazionale Galileo), located at "El Roque de los Muchachos" Observatory on La Palma, Spain. We combine our near-infrared spectrum with previously published data, and compare all the available spectra of this asteroid with the spectra of meteorites to constrain the mineralogy of the asteroid.

Results. Our spectrum of FG3 differs remarkably from previously published ones. Spectral classification performed using the complete visible and near-infrared range yields more than one result, varying from $\mathrm{C}$ to $\mathrm{Xk}$ types. However, all the possible spectral types indicate that this asteroid is a primitive object. The comparison with meteorites behaves in the same way, providing several good matches to our new near-infrared spectrum (CM2 carbonaceous chondrite, and L6 and H4 ordinary chondrites), and only one match in the case of the previously published spectra (weakly shocked $\mathrm{H} 4$ ordinary chondrite, dark vein). The albedo of the asteroid ( $\sim 0.04)$, is typical of a primitive object, and is consistent with the reflectance value at $0.55 \mu \mathrm{m}$ of the $\mathrm{CM} 2$ carbonaceous chondrite. Further observations will be essential to help us characterise more clearly the mineralogy of this asteroid.
\end{abstract}

Key words. minor planets, asteroids: individual: (175706) 1996 FG3 - methods: observational - techniques: spectroscopic

\section{Introduction}

Near-Earth asteroid (175706) $1996 \mathrm{FG}_{3}$ (hereafter FG3) was discovered on March 24, 1996, by R. H. McNaught from the Siding Spring Observatory, New South Wales, Australia. The asteroid was classified first by Whiteley \& Tholen (1999) and later by Binzel et al. (2004a) as a C-type. Dynamically, this is an Apollo asteroid with semimajor axis $a=1.054 \mathrm{AU}$, eccentricity $e=$ 0.35 , and inclination $i=1.98$ degrees. Only two years later, and using light-curve data from the Ondrejov Observatory, Pravec et al. (2000) discovered that the asteroid had a satellite. Mottola \& Lahulla (2000) observed mutual eclipse events in the system and determined the orbital and physical characteristics of the binary. Their best-fit solution for the orbit of the secondary around the primary was $a / D_{1}=(1.7 \pm 0.3)$, where $D_{1}$ is the diameter of the primary, and $e=0.05 \pm 0.05$. The diameters originally estimated for the primary and satellite were $\sim 1.4 \mathrm{~km}$ and $\sim 430 \mathrm{~m}$, respectively, assuming a geometric albedo typical of C-type asteroids (0.06). They also inferred that the primary body has normalised semiaxes of $A=1.05 \pm 0.02, B=0.95 \pm 0.02$, and $C=0.70 \pm 0.10$, hence is almost spherical. With an orbital period of $16.135 \pm 0.005 \mathrm{~h}$, a primary bulk density of $1.4 \pm 0.3 \mathrm{~g} / \mathrm{cm}^{3}$ and a mass of $2.1 \times 10^{12} \mathrm{~kg}$ were estimated. Measurements of the albedo derived from thermal infrared observations give a value of $p_{V}=0.042_{-0.017}^{+0.035}$, and a combined diameter of $D=1.84_{0.47}^{0.56} \mathrm{~km}$ (Mueller et al. 2011).

The nearly spherical shape and short rotation period $(3.6 \mathrm{~h})$ of FG3's primary provides some evidence of the binary's formation mechanism. Richardson et al. (1998) showed that NEOs with rubble-pile-like internal structures making slow, close encounters with the Earth or Venus are likely to undergo tidal disruption, and thus, objects on low-velocity encounters with the Earth, as is the case for FG3, can frequently be transformed into binary asteroids. However, some recent studies have shown that tidal disruption is not a very efficient binary formation mechanism, accounting for approximately $1-2 \%$ of NEAs being binaries, while the most likely observed fraction of binary NEAs 
is about $15 \%$ (Walsh \& Richardson 2006, 2008). Therefore, there must be other formation mechanisms that contribute significantly to this population. Walsh et al. (2008) demonstrated that small binary systems, formed by rapidly rotating primaries (typically under $10 \mathrm{~km}$ ) and one or more satellites, as in the case of FG3, are created by the slow spin up of a rubble pile asteroid by means of the thermal YORP effect.

Asteroid 1996 FG3 was identified as a good target for a space mission, as its orbit presents a $\Delta V$ of only $5.16 \mathrm{~km} \mathrm{~s}^{-1}$ (Perozzi et al. 2001; Christou 2003; Binzel et al. 2004b). Its primitive physical and binary nature also makes this asteroid an interesting target for a scientific rendezvous mission, thus it was chosen as a primary target for the new ESA Marco Polo-R mission, selected for the assessment study phase of ESA M3 missions. This will be the first sample return mission to study a primitive binary asteroid. Primitive asteroids are believed to consist of carbon-rich and organic material that have not been altered by processes such as melting and mixing that occurred during the early stages of the Solar System formation. Therefore, the Marco Polo-R mission will provide clues about the chemical composition and physical nature of the building blocks of terrestrial planets, as well as the nature and origin of organic materials. The binary nature of the target will allow more precise measurements of mass, gravity, and density than for a single object, as well as additional insights into the geology and geophysics of the system. Here we present a near-infrared reflectance spectrum of FG3. We compare our results with previously obtained near-infrared spectra of the asteroid, and compare with meteorite spectra to search for the closest analogue and constrain the mineralogy of this NEA.

\section{Observations}

Low resolution near-infrared spectra of asteroid 1996 FG3 was obtained on January 9, 2011, with the $3.6 \mathrm{~m}$ Telescopio Nazionale Galileo (TNG) using the low resolution mode of NICS (Near Infrared Camera Spectrograph). NICS is a multimode instrument based on a HgCdTe Hawaii $1024 \times 1024$ array. All spectroscopic modes use the large field camera, which has a plate scale of $0.25^{\prime \prime} /$ pixel (field of view of $4.2^{\prime} \times 4.2^{\prime}$ ). A $1.0^{\prime \prime}$ width slit, corresponding to a spectral resolving power of $R \sim 50$ that is quasi-constant along the spectrum, and the Amici prism disperser were used, covering the $0.8-2.5 \mu \mathrm{m}$ spectral range. The slit was oriented along the parallactic angle and the tracking was performed at the asteroid's proper motion. The acquisition consisted of two series of short exposure images offsetting the object between positions $A$ and $B$ in the slit direction. This process was repeated and a number of $A B B A$ cycles were acquired, with a total on-object exposure time of $1440 \mathrm{~s}$. To obtain the asteroid's reflectance spectrum, we observed three solar analogue stars from the Landolt catalogue (Landolt 1992) at similar airmass as the asteroid: SA 98-978, SA 102-1081, and SA 107-998. The reduction procedure followed de León et al. (2010b). After a standard bias and flat field correction, we subtracted consecutive $A$ and $B$ exposures from each $A B B A$ cycle, obtaining individual images from which $1 \mathrm{D}$ spectra were extracted and wavelength calibrated. Those individual spectra were then averaged and the result was divided by the individual spectrum of each solar analogue star. The resulting spectra were finally averaged and the final reflectance spectrum (Fig. 1, in red) was normalised to unity at $1.6 \mu \mathrm{m}$.

Another two near-infrared spectra were previously obtained by R. Binzel in the framework of the MIT-UH-IRTF (MINUS)
Table 1. Observational parameters for FG3's near-infrared spectra.

\begin{tabular}{lcccccc}
\hline \hline Source & Date & $\begin{array}{c}r \\
(\mathrm{AU})\end{array}$ & $\begin{array}{c}\Delta \\
(\mathrm{AU})\end{array}$ & $\begin{array}{c}\alpha \\
\left({ }^{\circ}\right)\end{array}$ & $m_{V}$ & $\begin{array}{c}\xi \\
\left({ }^{\circ}\right)\end{array}$ \\
\hline MINUS & $30 / 03 / 2009$ & 1.223 & 0.227 & 8.2 & 16.0 & 104 \\
MINUS & $27 / 04 / 2009$ & 1.080 & 0.158 & 58.6 & 16.5 & 104 \\
This work & $09 / 01 / 2011$ & 1.355 & 0.417 & 22.5 & 18.0 & 89 \\
\hline
\end{tabular}

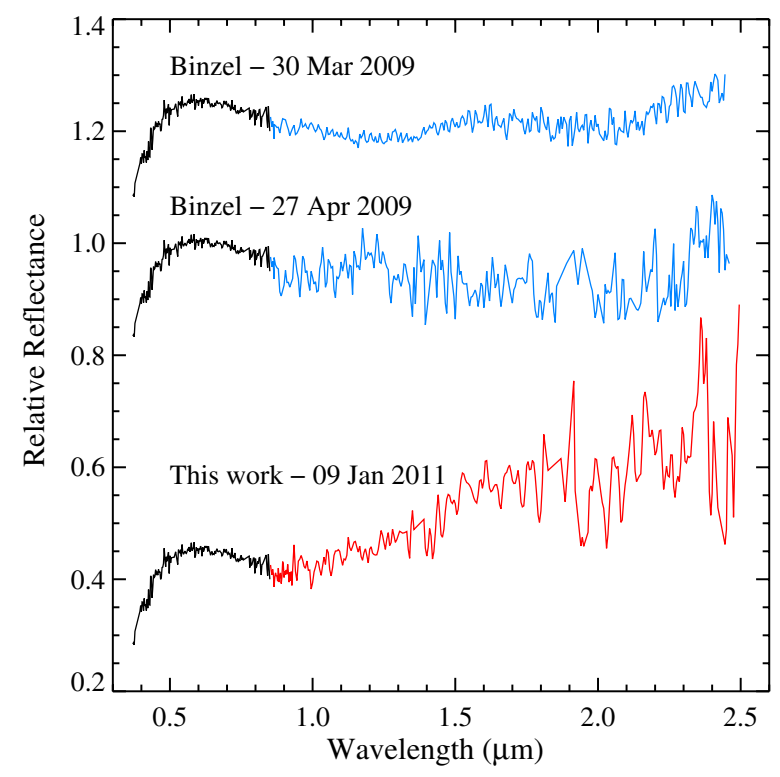

Fig. 1. Visible and near-infrared spectra of asteroid FG3. Visible spectrum has been taken from Binzel et al. (2001). Previous near-infrared spectra were obtained as part of the MIT-UH-IRTF survey, and are shown in blue. The near-infrared spectrum presented in this work is shown in red.

survey ${ }^{1}$, and are shown in Fig. 1 (blue lines). The spectra were taken in 2009, using SpeX, the low- to medium-resolution nearinfrared spectrograph and imager (Rayner et al. 2003), on the 3 m NASA IRTF located on Mauna Kea, Hawaii. The observational properties of these spectra as well as our near-infrared spectrum are shown in Table 1, and include the data source, date of observation, distance to both the Sun $(r)$ and the Earth $(\Delta)$, the phase angle $(\alpha)$, the visual magnitude $\left(m_{V}\right)$, and the aspect angle $(\xi)$. The aspect angle was computed considering the latest pole solution for FG3 (Scheirich \& Pravec 2009), and indicates that the three observations were taken when the asteroid was in near equatorial aspect. Nevertheless, the near-infrared spectra are quite different, as we discuss in the following sections.

\section{Spectral analysis}

We used the visible spectrum of FG3 from Binzel et al. (2001) to merge it with the three near-infrared spectra that we have, using the common interval $0.8-0.92 \mu \mathrm{m}$. We note how well the spectra fit in this interval. The complete spectra of the asteroid can be seen in Fig. 1. According to its visible spectrum, FG3 is classified as a C-type asteroid. We again classified the asteroid using the complete wavelength range and used the implementation of the Bus-DeMeo taxonomy (DeMeo et al. 2009) available on-line ${ }^{2}$.

\footnotetext{
1 http://smass.mit.edu/minus.html

2 http://smass.mit.edu/cgi-bin/busdemeoclass-cgi
} 
The first near-infrared spectrum (Binzel - 30 March 2009) does not provide a unique classification, which can be a C-type $(\mathrm{rms}=0.105)$, a Ch-type $(\mathrm{rms}=0.085)$, or an Xk-type $(\mathrm{rms}=0.234)$, and visual inspection is needed. The spectrum shows neither the absorption band centred at $0.7 \mu \mathrm{m}$ typical of Ch-type asteroids nor the redder spectral slope of Xk-types. Furthermore, the change in slope around 1.5-1.6 $\mu \mathrm{m}$ allows two potential absorption bands, centred at 1.22 and $1.97 \mu \mathrm{m}$. One could be tempted to associate these absorption bands, in particular the second one, with the presence of silicates, which would be indicative of the presence of pyroxene. However the depth, width, and centre position of the first absorption band are quite far from the values commonly computed for the absorption bands associated with silicates (both pyroxenes and olivines), so we rule out this possibility.

The second near-infrared spectrum (Binzel - 27 April 2009) does not show this change in slope around 1.5-1.6 $\mu \mathrm{m}$ and it is classified as a B-type asteroid $(\mathrm{rms}=0.064)$. We note the lower signal-to-noise ratio $(\mathrm{S} / \mathrm{N})$ of this spectrum, despite the asteroid being only half a visible magnitude fainter than the previous observation. Unfortunately, we have information about neither the exposure time nor the solar analogues used to obtain both spectra.

For the third near-infrared spectrum (ours), we are also unable to derive a unique classification. It might be either a Ch-type $(\mathrm{rms}=0.041)$ or an Xk-type $(\mathrm{rms}=0.127)$, and again visual inspection is needed. However, in this case we see a clear absorption band around $1 \mu \mathrm{m}$. Its wavelength position $(0.925 \mu \mathrm{m})$ is shifted to shorter and longer wavelengths than Ctype and Xk-type asteroids, respectively.

The taxonomical classification performed using the visible and near-infrared spectra of the asteroid gives in all cases a primitive type (C, B, or X). As mentioned in Sect. 1, recent measurements of the albedo of FG3 (Mueller et al. 2011) give a value of $p_{V}=0.042_{-0.017}^{+0.035}$, which is typical of primitive asteroids and indicates that the C-type is the most suitable classification.

\section{Meteorite comparison}

To obtain a broader view of the possible surface composition of FG3, we compared its visible and near-infrared spectra with all low-resolution visible and near-infrared meteorite spectra $(802$ meteorite samples) available from the RELAB public database (Pieters \& Hiroi 2004). In making these comparisons, we are aware of possible problems because the meteorite and asteroid spectra can be modified by the terrestrial and space weathering, respectively. We followed the method described in Mothé-Diniz \& Carvano (2005), which consisted of a first-order automated search, creating a list of "best matches", ordered according to the dispersion between the spectrum of the asteroid and that of the meteorite. We then plotted the spectrum of the asteroid against that of each meteorite in the list and inspected these plots visually. In this visual inspection, we searched for similarities in the shapes and positions of the $1 \mu \mathrm{m}$ absorption bands and their maxima, as well as similarities in the slope within the intervals $0.5-0.8$ and $1.5-2.5 \mu \mathrm{m}$. Both asteroid (red) and meteorite (blue) spectra are normalised to unity at $1.2 \mu \mathrm{m}$ (Fig. 2). We did this comparison for the spectrum with our near-infrared data and the highest $\mathrm{S} / \mathrm{N}$ spectrum of the two observations done by Binzel (30 March 2009).

In the case of our spectrum of FG3, the comparison yields several matches, containing both ordinary chondrites and carbonaceous chondrites, depending on which portion of the spectrum we focus on. Although we classified FG3 as C-type


Fig. 2. Best matches among meteorites from RELAB to the spectrum of FG3. The spectrum of the asteroid is shown in red, while the spectra of the meteorites/minerals are shown in blue: a) Meteorite Y-74662 (CM2), sample MP-TXH-011, grain size $<125 \mu \mathrm{m}$; b) Meteorite MacKinney (L4), sample MH-CMP-001-P1, powder; c) Meteorite Ozona (H5), sample MH-FPF-051-E, grain size $<150 \mu \mathrm{m}$; d) Meteorite Sete Lagoas (H4), sample MH-JFB-021, slab of a "dark vein”.

asteroid, its spectrum displays a clear " $1.0 \mu \mathrm{m}$ band", with a minimum just after $0.9 \mu \mathrm{m}(0.925 \pm 0.002 \mu \mathrm{m})$, that is not typical of C-type objects. The shape of both the first maximum and the $1 \mu \mathrm{m}$ band make the spectrum very similar to the spectra of some ordinary chondrites in the $0.4-1.4 \mu \mathrm{m}$ region (Fig. $2 \mathrm{~b}$ and c). In the near-infrared region, however, FG3 does not clearly exhibit the $2.0 \mu \mathrm{m}$ absorption band seen in the spectra of the majority of ordinary chondrites, which is associated with the presence of pyroxene. Even considering the higher noise in that region, which could easily obscure an absorption feature, the spectral slope from 1.5 to $2.5 \mu \mathrm{m}$ is incompatible with what is seen in the spectra of most ordinary chondrites. Although one can find some ordinary chondrites, specifically $\mathrm{H}$ chondrites, that yield a good match in that region, these fail to match the positions of both the centre and the first maximum of the $1 \mu \mathrm{m}$ absorption band (Fig. 2c). CM carbonaceous chondrites provide a good match in the region between 1.5 and $2.5 \mu \mathrm{m}$ (Fig. 2a), leading us back to the first classification of FG3 as a C-type. Although the $1.0 \mu \mathrm{m}$ band of CM meteorites have slightly different shapes and their centres are usually located at longer wavelengths, they provide the closest match to the spectrum of FG3 and are consistent with its spectral classification. Furthermore, the albedo of the asteroid ( 0.04) is similar to the reflectance of this CM2 meteorite sample at $0.55 \mu \mathrm{m}(0.047)$.

For the Binzel's spectrum of FG3, the comparison yields an excellent match, as seen in Fig. 2d. The spectrum of the meteorite was taken from a slab of the weakly shocked H4 ordinary chondrite Sete Lagoas (Keil et al. 1992). The sample corresponds to a "dark vein", an area covering both opaque melt vein and melt-breccia dike. According to the authors, "the spectrum is very similar to those of other shock veins, but has somewhat higher reflectance". The reflectance value of this meteorite sample at $0.55 \mu \mathrm{m}$, which can be compared to the albedo of an 
asteroid to a first approximation, is 0.091 , higher than the albedo of FG3 ( 0.04).

\section{Discussion}

All the near-infrared observations were made when the asteroid was in a near-equatorial aspect angle, and this, together with the small size of the primary, makes it difficult to explain any differences in the spectra in terms of observing different parts of the asteroid. Meteorite comparison provides also different results. In the case of our spectrum of FG3, and as discussed in the section above, the closest match corresponds to a CM2 carbonaceous chondrite, which is consistent with the taxonomical classification of the asteroid as a primitive object based on its visible spectrum, and with a reflectivity that is compatible with the albedo of the object. When we take Binzel's near-infrared spectrum (the one with highest $\mathrm{S} / \mathrm{N}$ ), we can identify one closest match, although the result unusually corresponds to a dark vein of a weakly shocked ordinary chondrite (Sete Lagoas). The formation of such veins by shocks involves shock-induced localized melting (Stöfer et al. 1991). In particular, melt dikes, which consist of opaque melt veins that have been intruded by shockproduced melt-breccia mixtures, occur when abundant shock melt is produced, typically by an impact. Melt veins and dikes make up only a small portion of the total shocked material in an impact crater, hence it is unlikely that the entire surface of this asteroid would be covered by this black ordinary chondrite material.

Nevertheless, caution must be taken when interpreting results by comparing meteorite and asteroid spectra. We know that different processes can alter the surface of the asteroids: the socalled "space weathering", which darkens and reddens their surfaces (Pieters et al. 2000), in particular for silicate-rich asteroids; the reddening of reflectance spectra (spectral slope) with increased phase angle (Murchie \& Pieters 1996); the size distribution of particles in the regolith, which affects the spectral slope and the absorption band depth; and changes in the temperature, which can alter the shape of spectral bands associated with olivines and pyroxenes (Singer \& Roush 1985).

\section{Conclusions}

We have presented new near-infrared data of the asteroid 1996 FG3, which is the primary target of the ESA Marco Polo-R mission. Our spectrum is quite different from those previously obtained in 2009 as part of the MIT-UH-IRTF survey. Although the observational conditions were not the same, in all cases the asteroid had a near equatorial aspect angle. Our spectral analysis performed using the complete visible and near-infrared wavelength range gives different taxonomical types, depending on which near-infrared data are considered, but in all cases the classification corresponds to primitive types $(\mathrm{C}, \mathrm{X}$, or $\mathrm{B})$.

Meteorite comparisons yield two different results. The closest match among meteorites to Binzel's spectrum of FG3 corresponds to a slab of a dark vein in a shocked ordinary chondrite (Sete Lagoas). The reflectance value at $0.55 \mu \mathrm{m}$ of the meteorite sample (0.091) is slightly higher than the albedo of the asteroid (0.04), within the uncertainties, and yet barely compatible with a primitive composition. Furthermore, it is very unlikely that dark melt veins and dikes cover a portion of the surface of the asteroid large enough to produce this spectrum. For our near-infrared spectrum, the closests matches among meteorites include a CM2 carbonaceous chondrite and two ordinary chondrites. A more detailed analysis of the spectrum of FG3 indicates that CM2 sample is the most likely match, which is consistent with a primitive composition and the albedo of the asteroid. This apparent difficulty to find a suitable meteorite analogue for a primitive asteroid, as is also the case of asteroid (21) Lutetia (Barucci et al. 2008; Vernazza et al. 2009; de León et al. 2011), illustrates the importance and the scientific significance of a space mission to study "in situ" the surface composition of this type of objects, and to get samples to be properly analysed in the laboratory.

As this asteroid is a primary target of a space mission, new observations will be extremely important to characterise this object and support data taken by the spacecraft. We will need not only visible and near-infrared spectra for mineralogical analysis, but also thermal and polarimetric observations to study its thermal inertia, particle surface distribution, and other surface properties.

Acknowledgements. This research has been partially funded by the Ministerio de Ciencia e Innovación through the project AYA 2009-08011. J.d.L. acknowledges a post-doctoral contract by the Junta de Andalucía research project PE07TIC2744. T.M.D. was supported by the Conselho Nacional de Desenvolvimento Científico e Tecnológico - $\mathrm{CNPq} /$ Brasil and by the Fundação de Amparo à Pesquisa do Estado do Rio de Janeiro - FAPERJ. J.L. gratefully acknowledges support from the Spanish "Ministerio de Ciencia e Innovación" project AYA2008-06202-C03-02. N.P.A. acknowledges the support from NASA Postdoctoral Program administered by Oak Ridge Associated Universities through a contract with NASA. H. Campins acknowledges support from NASA and the National Science Foundation. This paper is based on observations made with the Italian Telescopio Nazionale Galileo (TNG) operated on the island of La Palma by the Centro Galileo Galilei of the INAF (Instituto Nazionale di Astrofisica) at the Spanish Observatorio del Roque de los Muchachos of the Instituto de Astrofisica de Canarias.

\section{References}

Barucci, M. A., Fornasier, S., Dotto, E., et al. 2008, A\&A, 477, 665 Binzel, R. P., Harris, A. W., Bus, S. J., \& Burbine, T. H. 2001, Icarus, 151, 139 Binzel, R. P., Rivkin, A. S., Stuart, J. S., et al. 2004a, Icarus, 170, 259 Binzel, R. P., Perozzi, E., Rivkin, A. S., et al. 2004b, Meteor. Planet. Sci., 39, 351

Campins, H., Morbidelli, A., Tsiganis, K., et al. 2010, ApJ, 721, L53

Christou, A. A. 2003, Planet. Space Sci., 51, 221

Clark, B. E., Ziffer, J., Nervorny, D., et al. 2010, J. Geophys. Res., 115, 6005

de León, J., Campins, H., Tsiganis, K., et al. 2010a, A\&A, 513, A26

de León, J., Licandro, J., Serra-Ricart, M., et al. 2010b, A\&A, 517, A23

de León, J., Duffard, R., Lara, L. M., \& Lin, Z.-Y. 2011, A\&A, 527, A42

DeMeo, F. E., Binzel, R. P., Silvan, S. M., \& Bus, S. J. 2009, Icarus, 202, 160

Keil, K., Bell, J. F., \& Britt, D. 1992, Icarus, 98, 43

Landolt, A. U. 1992, AJ, 104, 340

Morbidelli, A., Gounelle, M., Levison, H. F., et al. 2006, Meteor. Planet. Sci., 41,875

Mothé-Diniz, T., \& Carvano, J. M. 2005, A\&A, 442, 727

Mottola, S., \& Lahulla, F. 2000, Icarus, 146, 556

Mueller, M., Delbó, M., Hora, J. L., et al. 2011, AJ, 141, 109

Murchie, S. L., \& Pieters, C. M. 1996, J. Geophys. Res., 101, 2201

Perozzi, E., Rossi, A., \& Valsecchi, G. B. 2001, Planet. Space Sci., 49, 3

Pieters, C. M., Taylor, L. A., Noble, S. K., et al. 2000, Meteor. Planet. Sci., 35, 1101

Pieters, C. M., \& Hiroi, T. 2004, in 35th Annual Lunar and Planetary Institute Conference Abstracts, 35, 1720

Pravec, P., Šarounová, L., Rabinowitz, D. L., et al. 2000, Icarus, 146, 190

Rayner, J. T., Toomey, D. W., Onaka, P. M., et al. 2003, PASP, 115, 362

Richardson, D. C., Bottke, W. F., \& Love, S. G. 1998, Icarus, 134, 47

Scheirich, P., \& Pravec, P. 2009, Icarus, 200, 531

Singer, R. B., \& Roush, T. L. 1985, J. Geophys. Res., 90, 12434

Stöffer, D., Keil, K., \& Scott, R. D. 1991, Geochim. Cosmochim. Acta, 55, 3845

Vernazza, P., Brunetto, R., Binzel, R. P., et al. 2009, Icarus, 202, 477

Walsh, K. J., \& Richardson, D. C. 2006, Icarus, 180, 201

Walsh, K. J., \& Richardson, D. C. 2008, Icarus, 193, 553

Walsh, K. J., Richardson, D. C., \& Michel, P. 2008, Nature, 454, L188

Whiteley, R. J., \& Tholen, D. J. 1999, in 31st Am. Astron. Soc. DPS Meeting, 11.02 